Article

\title{
Three-Dimensional Wind Field Construction and Wind Turbine Siting in an Urban Environment
}

\author{
Mingrui Liu and Xiuling Wang * \\ Mechanical and Civil Engineering Department, Purdue University Northwest, Hammond, IN 46323, USA; \\ liu2310@pnw.edu \\ * Correspondence: wangx@pnw.edu
}

Received: 1 July 2020; Accepted: 10 August 2020; Published: 16 August 2020

\begin{abstract}
Three-dimensional urban wind field construction plays an important role not only in the analysis of pedestrian levels of comfort but also in the effectiveness of harnessing wind energy in an urban environment. However, it is challenging to accurately simulate urban wind flow due to the complex land use in urban environments. In this study, a three-dimensional numerical model was developed for urban wind flow construction. To obtain an accurate urban wind field, various turbulence models, including the Reynolds stress model (RSM), $k-\omega$ shear stress transport (SST), realizable $k-\varepsilon$, and (Re-Normalisation Group (RNG) $k-\varepsilon$ models were tested. Simulation results were compared with experimental data in the literature. The RSM model showed promising potential in simulating urban wind flow. The model was then adopted to simulate urban wind flow for Purdue University Northwest, which is located in the Northwest Indiana urban region. Based on the simulation results, the optimal location was identified for urban wind turbine siting.
\end{abstract}

Keywords: three-dimensional wind field construction; urban wind environment; urban wind turbine siting

\section{Introduction}

Three-dimensional urban wind field construction is important for many activities: weather forecasting, natural ventilation system design, pedestrian comfort level analysis, atmospheric dispersion assessments, and urban wind energy harnessing. However, it is challenging to accurately simulate urban wind flow due to complex land usage and terrain conditions, as well as the variety of building shapes, heights, densities, and layout configurations in urban environments. Stathopoulos et al. provided some views on the potential benefits and challenges in urban wind energy [1]; they reviewed the urban wind energy knowledge and discussed some current issues. Recent progress that was made in urban wind energy assessment was also addressed.

Many researchers focus on urban wind field construction and analysis. The straightforward methods are wind tunnel testing and field measurements [2-5]. Earlier wind tunnel tests were done by Wiren et al. [2], where they conducted experimental studies of wind velocities in passages between and through block-type building models with various types of layouts. Later, Tsang et al. [3] focused on wind tunnel studies of a pedestrian-level wind environment around tall buildings. More recently, Weerasuriya et al. [4] conducted wind tunnel studies to uncover the effects of twisted wind flows on pedestrian-level wind fields in an urban environment. Finally, Li et al. performed wind tunnel studies and tried to find the correlation between urban space quantification and pedestrian-level ventilation [5].

With the rapid development of computational resource and meshing techniques, increasingly more researchers have used computational fluid dynamics along with measured wind tunnel data to simulate and analyze wind flow in an urban environment. Many of the simulations were conducted by using Reynolds-averaged Navier-Stokes (RANS) models or the large-eddy simulation (LES) approach. 
Blocken et al. [6] reviewed wind-tunnel and computational fluid dynamics (CFD) techniques to address their accuracy for wind comfort assessment. A comparison between the RANS model and LES for urban wind simulations was provided. Rodi used various $k-\varepsilon$ models, Reynolds stress models, and LES models for urban wind simulations [7], where the results were compared with experimental data. The performance, cost, and potential of solving complex flow problems using those turbulence models were also compared. Rodi pointed out that the use of RANS calculations underestimated the turbulence fluctuations in general, while the use of LES gave better simulation details of the turbulence flow with the cost of a large increase in computing time. In the LES model, the unresolved small-scale turbulent motion was modeled, which was less influenced by the boundary conditions. Johnson and Hunter simulated urban wind flow using the $k-\varepsilon$ model and revealed the broad flow features [8]. Compared with the wind tunnel experiments, a general agreement between the numerical results and experimental data was demonstrated. Willis investigated and tested the CFD validation guidelines in an urban wind simulation [9], while Song and He conducted a numerical simulation using an LES model when evaluating pedestrian wind flow [10]; in their studies, different geometry and wind conditions were adopted and simulation results were compared with wind tunnel experimental data.

Different variations of RANS models were widely used by many others for simulating urban wind flow: Santiago and Martilli used the standard $k-\varepsilon$ model when simulating wind flow over a regular array of cubes [11]; Zheng et al. conducted the pedestrian-level wind environment study via wind tunnel tests and realizable $k-\varepsilon$ simulations [12]; Gnatowska et al. used the realizable $k-\varepsilon$ model to simulate flow fields in an urban environment, where the results were compared with Particle Image Velocimetry (PIV) data in a wind tunnel and a detailed description of the velocity field in the built-up environment was obtained [13]; Akashi et al. employed the Re-Normalisation Group (RNG) $k-\varepsilon$ model to simulate the urban wind flow under different building layout effects [14]; Hu et al. applied the RNG $k-\varepsilon$ model during a quantitative investigation of the building density effects on residential wind environments [15]; and Liu et al. used the RNG $k-\varepsilon$ model to simulate the wind distribution in an urban community with a full-scale geometrical model coupled with a microscale model [16].

Among these modeling works, LES simulations can reproduce more accurate details of the turbulence flow, but because of its large computational cost, most researchers choose to use a RANS model for urban wind flow simulations instead. In urban wind flow analysis, understanding the flow features within the passages is more important than capturing the detailed turbulence features near wall regions. The RANS models satisfy these requirements and are more computationally affordable than an LES. Reiter compared the standard $k-\varepsilon$ model, realizable $k-\varepsilon$ model, and RSM model in urban wind simulations [17], but some other popular RANS models, such as the RNG $k-\varepsilon$ model and $k-\omega$ shear stress transport (SST) model were not included. The comparisons between different RANS models for urban wind flow simulations are limited in the literature.

There are two main parts to this paper. First, various popular RANS turbulence models, including the RNG $k-\varepsilon$ model, realizable $k-\varepsilon$ model, $k-\omega$ SST model, and RSM, were used to simulate wind flow in an urban environment. The simulation results were compared with wind tunnel experimental data. Among all those models, the RSM showed the most promising results for urban flow simulations, with the simulation results closely matching the experimental data. Second, wind turbine siting in an urban environment was investigated for Purdue University Northwest campus. By implementing the selected turbulence model coupled with a year-long wind data analysis, an optimal location was selected for a small vertical axis wind turbine (VAWT).

\section{Materials and Methods}

Two parts are presented here: the validation study of the various turbulence models adopted for urban wind field simulations and the urban wind flow analysis and turbine siting for Purdue University Northwest, which is located in Northwestern Indiana, a suburban environment. 


\subsection{Model Validation}

The three-dimensional CFD model was first developed to simulate urban wind flow around a single building and two building test cases. The buildings were configured to follow the wind tunnel experiments conducted by Wiren et al. [2].

For the case concerning wind flow around a single building, the detailed building configuration is shown in Figure 1a. The building had a length (L) of $80 \mathrm{~m}$, height (H) of $18 \mathrm{~m}$, and width (B) of $12 \mathrm{~m}$. At the pedestrian level, there is a pedestrian crossing with a length (B) of $12 \mathrm{~m}$, a width (b) of $6 \mathrm{~m}$, and a height (h) of $4 \mathrm{~m}$ in the center of the building.

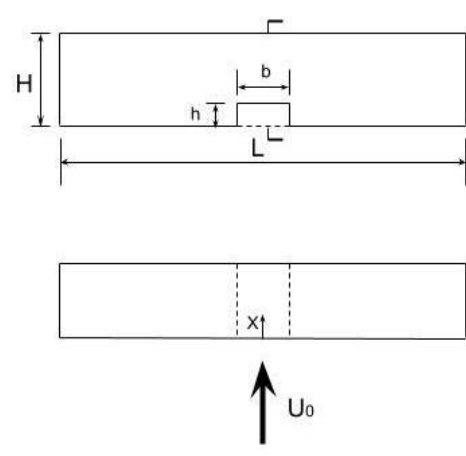

(a)

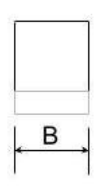

(b)

Figure 1. Configuration for the case concerning single-building wind flow validation: (a) building geometry and (b) computational domain.

These wind tunnel tests were measured at the height of $2 \mathrm{~m}$ at the middle line of the pedestrian channel. The computational domain size was $480 \mathrm{~m} \times 440 \mathrm{~m} \times 108 \mathrm{~m}$. To avoid boundary influences, the distance from the inlet to the front surface and all of the side surfaces of the building to the boundaries of the computational domain was $10 \mathrm{H}$. To make sure the flow reached a fully developed condition, the distance from the outlet to the back surface of the building was $16 \mathrm{H}$.

For the case concerning wind flow around two buildings, the building layout is shown in Figure 2. Two identical buildings were arranged perpendicular to each other. The buildings were separated by a passage with distance $\mathrm{b}$ is $4 \mathrm{~m}$. The wind was blowing perpendicular to the front line formed by the two buildings.

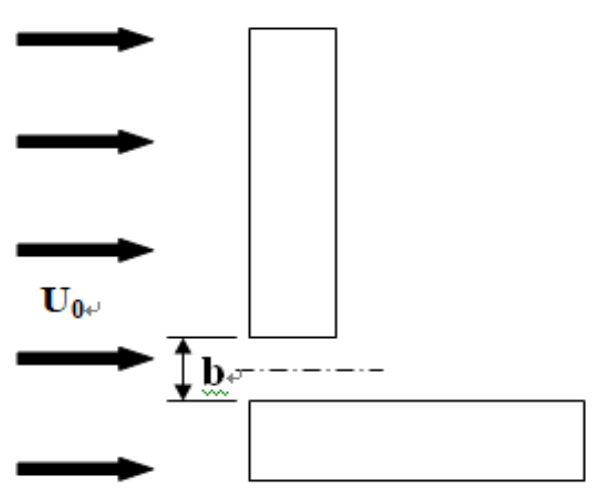

(a)

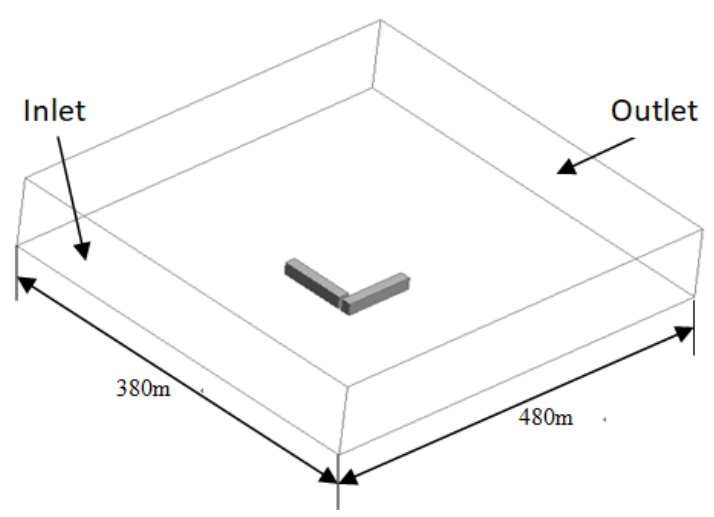

(b)

Figure 2. Configuration for the case concerning two-building wind flow validation: (a) building geometry and (b) computational domain. 
Similarly to the previous case, a sufficiently large computational domain was set up, with a height of $108 \mathrm{~m}$, a length of $480 \mathrm{~m}$, and a width of $380 \mathrm{~m}$. The distance from the inlet to the front surface of the building and the building side surfaces to the boundaries of the computational domain was $10 \mathrm{H}$. For this validation study, the downstream of the building was $16 \mathrm{H}$.

For the two validation cases, the inlet velocity was set up using the power law $\frac{U}{U_{0}}=\left(\frac{Z}{Z_{0}}\right)^{\alpha}$. Based on Wiren's wind tunnel experiments [2], $U_{0}(10 \mathrm{~m} / \mathrm{s})$ and $z_{0}(2 \mathrm{~m})$ were the reference velocity and height, respectively; $\alpha$ was 0.125 , and $U$ is the velocity at vertical distance $z$ from the surface. The pressure outlet was used as the outflow condition, the ground was set as a non-slip boundary condition, and symmetries were set up for all the side and top boundaries.

Different turbulence models were used in the two validation cases. Detailed information is given in the next session for the governing equations, as well as the comparison for the turbulence models adopted in this study.

\subsection{Turbulence Models}

The commercial software ANSYS ${ }^{\circledR}$ Fluent 19.1 (Ansys Inc., Canonsburg, PA, USA) was used for the simulations. Reynolds-averaged Navier-Stokes models (RANS models) were used to solve the transport equations of the airflow and simulate all turbulence scales. The RANS models are widely used in urban flow simulations because they yield relatively accurate simulation results and are computationally efficient compared with LES models [18]. In this study, various RANS models, including realizable $k-\varepsilon$, RNG $k-\varepsilon, k-\omega$ SST, and RSM, were implemented to solve the two validation cases. Brief descriptions of each turbulence model are listed below.

The compressible RANS equation for air can be written in the form of a Cartesian tensor using the summation convention:

$$
\begin{gathered}
\frac{\partial \rho}{\partial t}+\frac{\partial}{\partial x_{i}}\left(\rho u_{i}\right)=0 \\
\left(\rho u_{i}\right)+\frac{\partial}{\partial x_{j}}\left(\rho u_{i} u_{j}\right)=-\frac{\partial p}{\partial x_{i}}+\frac{\partial \sigma_{i j}}{\partial x_{j}}+\frac{\partial}{\partial x_{j}}\left(-\rho u_{i}^{\prime} u_{j}^{\prime}\right) .
\end{gathered}
$$

The $k-\varepsilon$ models assume that the turbulence viscosity is linked to the turbulence kinetic energy and turbulent frequency via the relation:

$$
\mu_{t}=\rho \frac{k}{\omega}
$$

In this research, turbulence flow was modeled using the realizable $k-\varepsilon$ model, RNG $k$ - $\varepsilon$ model, $\mathrm{k}-\omega$ SST model, and Reynolds stress model (RSM) for airflow to compute the turbulent kinetic energy $(k)$ and the turbulent kinetic energy dissipation $(\varepsilon)$.

The exact $k-\varepsilon$ equations have many terms that have not yet been calculated. In order to facilitate understanding and calculations, the standard $k-\varepsilon$ model was proposed by Launder and Spalding, which can be used as a basic method that is applied to engineering flow field calculations. The kinetic energy and turbulence dissipation rates in this model are described as:

$$
\begin{gathered}
\frac{\partial}{\partial t}(\rho k)+\frac{\partial}{\partial x_{i}}\left(\rho k u_{i}\right)=\frac{\partial}{\partial x_{j}}\left[\left(\mu+\frac{\mu_{t}}{\sigma_{k}}\right) \frac{\partial k}{\partial x_{j}}\right]+P_{k}+P_{b}+S_{k}-\rho \varepsilon-Y_{M}, \\
\frac{\partial}{\partial t}(\rho \varepsilon)+\frac{\partial}{\partial x_{i}}\left(\rho \varepsilon u_{i}\right)=\frac{\partial}{\partial x_{j}}\left[\left(\mu+\frac{\mu_{t}}{\sigma_{k}}\right) \frac{\partial k}{\partial x_{j}}\right]+C_{1 \varepsilon} \frac{\varepsilon}{k}\left(G_{k}+C_{3 \varepsilon} G_{b}\right)-C_{2 \varepsilon} \rho \frac{\varepsilon^{2}}{k}+S_{\varepsilon},
\end{gathered}
$$

where $u_{i}$ is the velocity component in the corresponding direction and $\mu_{t}$ is the turbulent viscosity computed using $k$ and $\varepsilon$ as follows:

$$
\mu_{t}=\rho C_{\mu} \frac{k^{2}}{\varepsilon}
$$

where $C_{\mu}, C_{1 \varepsilon}, C_{2 \varepsilon}, \sigma_{k}$, and $\sigma_{\varepsilon}$ are constants, which are $0.09,1.44,1.92,1.00$, and 1.30, respectively. 
The realizable $k-\varepsilon$ model adds a formula to the turbulent viscosity and adds a new transport equation to the dissipation rate, such that the expressions of $k$ and $\varepsilon$ are:

$$
\begin{gathered}
\frac{\partial}{\partial t}(\rho k)+\frac{\partial}{\partial x_{i}}\left(\rho k u_{i}\right)=\frac{\partial}{\partial x_{j}}\left[\left(\mu+\frac{\mu_{t}}{\sigma_{k}}\right) \frac{\partial k}{\partial x_{j}}\right]+P_{k}+P_{b}+S_{k}-\rho \varepsilon-Y_{M}, \\
\frac{\partial}{\partial t}(\rho \varepsilon)+\frac{\partial}{\partial x_{j}}\left(\rho \varepsilon u_{j}\right)=\frac{\partial}{\partial x_{j}}\left[\left(\mu+\frac{\mu_{t}}{\sigma_{k}}\right) \frac{\partial k}{\partial x_{j}}\right]+\rho C_{1} S_{\varepsilon}+\rho C_{2} \frac{\varepsilon^{2}}{k+\sqrt{v \varepsilon}}+C_{1 \varepsilon} \frac{\varepsilon}{k}+C_{3 \varepsilon} G_{b}+S_{\varepsilon},
\end{gathered}
$$

where:

$$
\begin{gathered}
C_{1}=\max \left[0.43, \frac{\eta}{\eta+5}\right], \\
\eta=S \frac{k}{\varepsilon} \\
S=\sqrt{2 S_{i j} S_{i j}},
\end{gathered}
$$

The RNG $k-\varepsilon$ model was derived from a strict statistical mathematical technique to solve the problem for the effect of a smaller Reynolds number flow viscosity. The RNG and realizable $k-\varepsilon$ models have shown significant improvements over the standard $k-\varepsilon$ model in terms of the flow characteristics, including strong streamline curvature, vortex, and rotation [19]. The equations for $k$ and $\varepsilon$ are:

$$
\begin{gathered}
\frac{\partial}{\partial t}(\rho k)+\frac{\partial}{\partial x_{i}}\left(\rho k u_{i}\right)=\frac{\partial}{\partial x_{j}}\left[\left(\mu+\frac{\mu_{t}}{\sigma_{k}}\right) \frac{\partial k}{\partial x_{j}}\right]+P_{k}-\rho \varepsilon \\
\frac{\partial}{\partial t}(\rho \varepsilon)+\frac{\partial}{\partial x_{i}}\left(\rho \varepsilon u_{i}\right)=\frac{\partial}{\partial x_{j}}\left[\left(\mu+\frac{\mu_{t}}{\sigma_{\varepsilon}}\right) \frac{\partial k}{\partial x_{j}}\right]+C_{1 \varepsilon} \frac{\varepsilon}{k} P_{k}+C_{2 \varepsilon}^{*} \frac{\varepsilon}{k} P_{k}
\end{gathered}
$$

where:

$$
C_{2 \varepsilon}^{*}=C_{2 \varepsilon}+\frac{C_{\mu} \eta^{3}\left(1-\eta / \eta_{0}\right)}{1+\beta \eta^{3}}, \eta_{0}=4.38
$$

The $k$ - $\omega$ shear stress transport (SST) is a two-equation eddy-viscosity model. The inner parts of the boundary layer are solved using the $k-\omega$ model, while the $k-\varepsilon$ model was implemented in the free shear flow. This model shows good behavior in adverse pressure gradients and separating flow regions. Menter [20] discussed this method in detail. The appropriate transport behavior can be obtained by formulating the vortex viscosity as follows:

$$
v_{t}=\frac{a_{1} k}{\max \left(a_{1} \omega_{r} S F_{2}\right)} .
$$

Note that the production term of $\omega$ is given by:

$$
P_{\omega}=\left(\frac{a_{3}}{v_{t}}\right) .
$$

This formulation differs from the standard $k-\varepsilon$ model. The kinetic energy and the specific dissipation rate equations are:

$$
\begin{gathered}
\frac{\partial k}{\partial t}+U_{j} \frac{\partial k}{\partial x_{j}}=\frac{\partial}{\partial x_{j}}\left[\left(v+\sigma_{k} v_{T}\right) \frac{\partial k}{\partial x_{j}}\right]+P_{k}-\beta^{*} k \omega \\
\frac{\partial \omega}{\partial t}+U_{j} \frac{\partial \omega}{\partial x_{j}}=\alpha S^{2}-\beta \omega^{2}+\frac{\partial}{\partial x_{j}}\left[\left(v+\sigma_{\omega} v_{T}\right) \frac{\partial \omega}{\partial x_{j}}\right]+2\left(1-F_{1}\right) \sigma_{\omega 2} \frac{1}{\omega} \frac{\partial k}{\partial x_{j}} \frac{\partial \omega}{\partial x_{j}}
\end{gathered}
$$


where:

$$
\begin{gathered}
P_{k}=\min \left(\tau_{i j} \frac{\partial U_{i}}{\partial x_{j}}, 10 \beta^{*} k \omega\right), \\
F_{1}=\tanh \left\{\left\{\min \left[\max \left(\frac{\sqrt{k}}{\beta^{*} \omega y}, \frac{500 v}{y^{2} \omega}\right), \frac{4 \sigma_{\omega 2}}{C D_{k \omega y^{2}}}\right]\right\}^{4}\right\},
\end{gathered}
$$

The Reynolds stress model (RSM), known as the Reynolds stress transport model, represents the most complete classical turbulence model. The method of closure used is usually called a second-order closure. This model can explain the complex conditions in turbulent flow and the Reynolds stress's effects. The governing equations for the transport and momentum are written as follows:

$$
\begin{gathered}
\frac{\partial \rho \underline{U_{i} U_{j}}}{\partial t}+\frac{\partial k}{\partial x_{k}}\left(U_{k} \rho \underline{U_{i} U_{j}}\right)-\frac{\partial}{\partial x_{k}}\left[\left(\delta_{k l} \mu+\rho C_{S} \frac{k}{\varepsilon} \frac{\left.U_{k} U_{l}\right)}{\partial x_{l}}\right]=P_{i j}-\frac{2}{3} \delta_{i j} \rho \varepsilon+\varnothing_{i j}+P_{i j, b},\right. \\
\frac{\partial \rho U_{i}}{\partial t}+\frac{\partial}{\partial x_{j}}\left(\rho U_{i} U_{j}\right)-\frac{\partial}{\partial x_{j}}\left[\mu\left(\frac{\partial \rho U_{i}}{\partial x_{j}}+\frac{\partial \rho U_{j}}{\partial x_{i}}\right)\right]=-\frac{\partial p^{\prime \prime}}{\partial x_{i}}-\frac{\partial}{\partial x_{j}}\left(\rho \underline{u_{i} u_{j}}\right)+S_{M_{i},} \\
p^{\prime \prime}=p+\frac{2}{3} \mu \frac{\partial k}{\partial x_{k}} .
\end{gathered}
$$

The transport equations of the RSM can be written as [19]:

$$
\begin{aligned}
& \frac{\partial \rho \underline{U_{i} U_{j}}}{\partial t}+\frac{\partial k}{\partial x_{k}}\left(U_{k} \rho \underline{\left.U_{i} U_{j}\right)}\right)=\frac{\partial}{\partial x_{k}} {\left[\left(\rho \underline{U_{i}^{\prime} U_{j}^{\prime} U_{k}^{\prime}}+\underline{\rho^{\prime}\left(\delta_{k j} U_{i}^{\prime}+\delta_{k j} U_{i}^{\prime}\right)}\right)\right]+\frac{\partial}{\partial x_{k}}\left[\mu \frac{\partial}{\partial x_{k}}\left(\underline{U_{i}^{\prime} U_{j}^{\prime}}\right)\right] } \\
&-\rho\left(\underline{U_{i}^{\prime} U_{k}^{\prime}} \frac{\partial u_{j}}{\partial x_{k}}+\underline{U_{j}^{\prime} U_{k}^{\prime} \frac{\partial u_{i}}{\partial x_{k}}}\right)-\rho \beta\left(\underline{\left.g_{i} U_{j}^{\prime} \theta+g_{j} \underline{U_{i}^{\prime} \theta}\right)+P^{\prime}\left(\frac{\partial u_{i}^{\prime}}{\partial x_{j}}+\frac{u_{j}^{\prime}}{\partial x_{i}}\right)}\right. \\
&-2 \mu \frac{\partial u_{i}^{\prime} \partial u_{j}^{\prime}}{\partial x_{k}}-2 \rho \Omega_{k}\left(\underline{U_{j}^{\prime} U_{m}^{\prime} \varepsilon_{i k m}}+\underline{U_{i}^{\prime} U_{m}^{\prime} \varepsilon_{j k m}}\right)+S_{\text {user }}
\end{aligned}
$$

\subsection{Urban Wind Turbine Siting}

Purdue University Northwest (PNW) is located in Northwest Indiana, a suburban environment. An urban wind flow simulation for PNW was conducted to help identify the optimal location of a VAWT on campus.

Figure 3a shows the top view of the campus environment from Google Maps and Figure 3b shows the layout of the campus's main buildings. The main buildings include the Student Union and Library Building (SULB), Gyte building, Porter Hall, Parking Garage building, Power building, Potter building, Classroom Office building (CLO), and Anderson building. All the main buildings were included in the three-dimensional urban wind field geometry models. To reduce the complexity of meshing and further computational efforts, some detailed building structures were neglected.

The computational domain was $740 \mathrm{~m}$ in length, $630 \mathrm{~m}$ in width, and $100 \mathrm{~m}$ in height. It was selected to avoid the boundary effects around the inflow and sides, and to make sure the downstream reached a fully developed flow region. The distance from the inlet to the SULB front surface was $8 \mathrm{H}, 16 \mathrm{H}$ downstream, $5 \mathrm{H}$ on each building side, and about $5 \mathrm{H}$ above the building's roof. The tallest building height $(\mathrm{H})$ was $17.27 \mathrm{~m}$. 


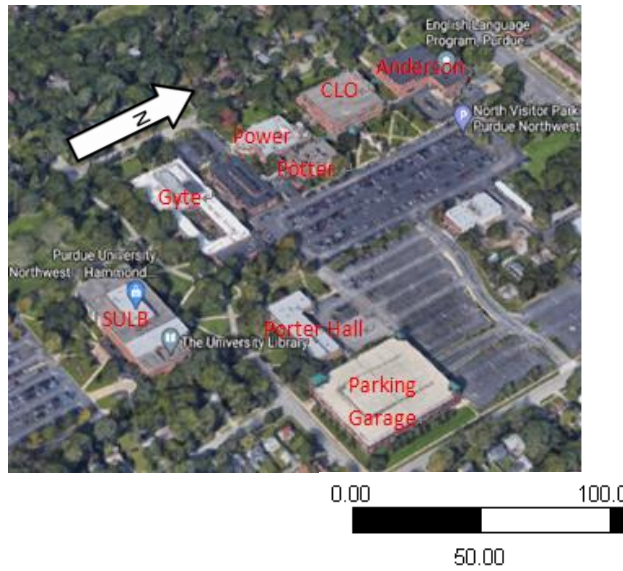

(a)

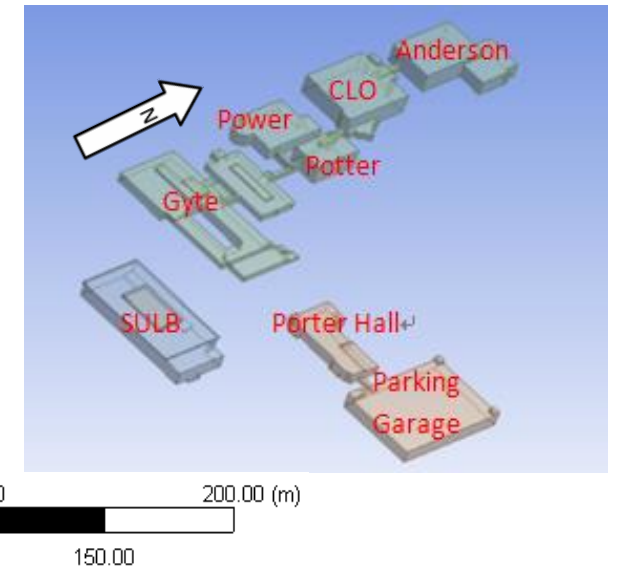

(b)

Figure 3. Overview of the Purdue University Northwest (PNW) campus buildings: (a) top view from Google Maps and (b) the building layout 3-D model. CLO: Classroom Office Building, SULB: Student Union and Library Building.

To monitor the on-campus wind flow conditions, three wind sensors were installed on the roof of the SULB. The height of the SULB was $16.43 \mathrm{~m}$, the location of each data sensor is shown in Figure 4.

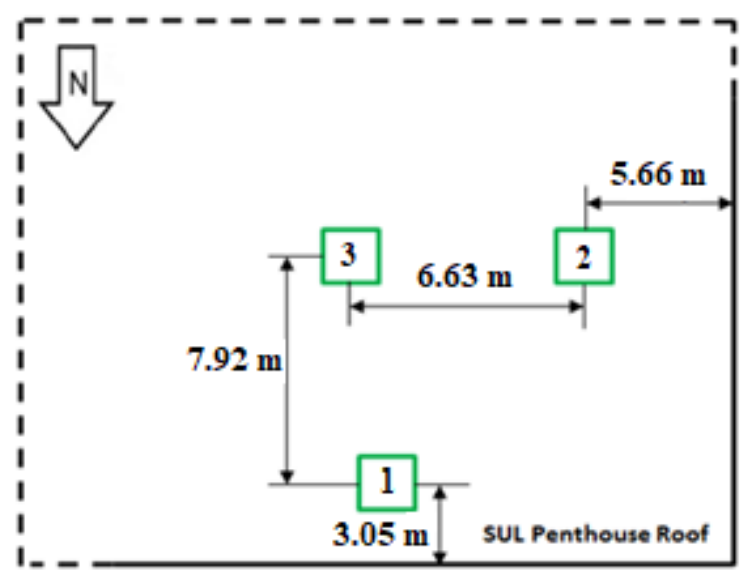

Figure 4. Location of three wind data sensors.

The boundary conditions applied in the PNW campus wind flow simulation are listed in Table 1.

Table 1. Boundary conditions.

\begin{tabular}{ccc}
\hline Zone & Boundary Type & Profile \\
\hline Inlet & Velocity inlet & User-defined function \\
\hline Outlet & Pressure outlet & $\begin{array}{r}\text { Backflow turbulent intensity (\%): } 5 \\
\text { Backflow turbulent viscosity ratio: } 10\end{array}$ \\
\hline Top & Symmetry & N/A \\
\hline bottom & No-slip wall & $\begin{array}{c}\text { Roughness height: } 0 \\
\text { Roughness constant: } 0.5\end{array}$ \\
\hline Sides & Pressure outlet & $\begin{array}{c}\text { Backflow turbulent intensity (\%): } 5 \\
\text { Backflow turbulent viscosity ratio: } 10\end{array}$ \\
\hline Building surface & No-slip wall & $\begin{array}{c}\text { Roughness height: } 0 \\
\text { Roughness constant: } 0.5\end{array}$ \\
\hline
\end{tabular}


The prevailing wind speed and wind direction were determined based on an analysis of year-long wind data [21] from the three data sensors. Recorded wind data from April 2010 to April 2011 were used. After the prevailing wind information was determined, the inflow wind profile was set up, with the details being covered in Section 3.2.

The outlet was a pressure outlet and the other two sides of the domain were also regarded as pressure outlets. The ground was the bottom of the computational domain and was therefore treated as a no-slip wall. The building walls were considered to be smooth no-slip wall boundary conditions. The top boundary of the computational domain was set as a symmetry condition.

\subsection{Statistical Analysis}

To evaluate the wind energy potential at a given site, statistical analysis can be used. The probability distributions and statistical techniques become especially important if only summary data are available. There are two commonly used probability density functions for wind data analysis: the Weibull distribution and the Rayleigh distribution.

The Weibull probability density function is based on two parameters: $k$ and $c$, with $k$ being a shape factor, while $c$ is a scale factor. Both $k$ and $c$ are functions of the wind speed standard deviation and the average wind velocity. It is not straightforward to obtain $k$ and $c$. There are several different ways to do the calculation. In this study, the empirical method was used to calculate $k$ and $c$ :

$$
\begin{gathered}
k=\left(\frac{\sigma}{V_{a v g}}\right)^{-1.086}, \\
c=\frac{V_{a v g}}{\Gamma\left(1+\frac{1}{k}\right)} .
\end{gathered}
$$

The average wind velocity is calculated as:

$$
V_{\text {avg }}=c \Gamma\left(1+\frac{1}{k}\right)
$$

where the $\Gamma(x)$ is the gamma function, which is defined as $\Gamma(x)=\int_{0}^{\infty} e^{-t} t^{x-1} d t$ and it can be calculated using the recursion formula:

$$
\Gamma(x+1)=x \Gamma(x) .
$$

In the Weibull distribution, a higher value of $k$ shows a sharper peak of the probability density function curve, indicating that the wind speed variation is less; on the other hand, a lower value of $k$ indicates a bigger variability about the mean wind speed. Generally, the sites with low shape parameters and high mean wind speeds will make them good candidates for wind turbine siting since these features usually indicate that those sites have the highest total mean power density [22]. The Weibull probability density function is defined as:

$$
P\left(V_{w}\right)=\left(\frac{k}{c}\right)\left(\frac{V_{w}}{c}\right)^{k-1} \exp \left[-\left(\frac{V_{w}}{c}\right)^{k}\right] .
$$

Compared to the Weibull distribution, the Rayleigh distribution is simpler as it only requires the knowledge of the mean wind speed. The probability density function for a Rayleigh distribution is given as:

$$
P\left(V_{w}\right)=\frac{\pi}{2}\left(\frac{V_{w}}{V_{a v g}{ }^{2}}\right) \exp \left[-\frac{\pi}{4}\left(\frac{V_{w}}{V_{a v g}}\right)^{2}\right] .
$$

In the Rayleigh distribution, the larger value of the mean wind speed indicates a higher probability at higher wind speeds for specific sites. Sites with a higher mean wind speed are usually associated 
with good wind energy potential. The two parameters found in the Weibull distribution can better represent a wider variety of wind regimes [23].

\section{Results}

\subsection{Turbulence Model Selection}

To compare the performance of different turbulence models when simulating urban wind flow, different turbulence models were adopted for urban wind flow simulations in both the single-building and two-building cases. The tested turbulence models were the RNG $k-\varepsilon$, realizable $k-\varepsilon$ model, $k-\omega$ SST model, and second-order RSM. The simulation results were compared with experimental results from wind tunnel tests by Wiren et al. [2].

\subsubsection{Validation for Wind in the Single-Building Case}

Figure $5 \mathrm{a}$ shows the meshes for the computational domain and Figure $5 \mathrm{~b}$ shows the zoomed-in view of the meshes along the building. The boundary layer meshes that were generated are noticeable, along with the fine meshes around the building for enhanced capturing of the boundary layer flow features. Three body of influence regions were selected: around the pedestrian channel with a mesh size of $0.5 \mathrm{~m}$, around the building with a mesh size of $1 \mathrm{~m}$, and another near the upstream and downstream region with a mesh size of $2 \mathrm{~m}$. The rest of the computational domain mesh size was $10 \mathrm{~m}$. In total, 10 inflation layers were used with a 1.2 growth rate. A mesh-independent study was conducted. The medium-sized mesh was adopted in the simulation for different turbulence models as it brought a good balance between the computational accuracy and cost. The total number of elements used in the final simulation for the medium mesh was 2,189,837.

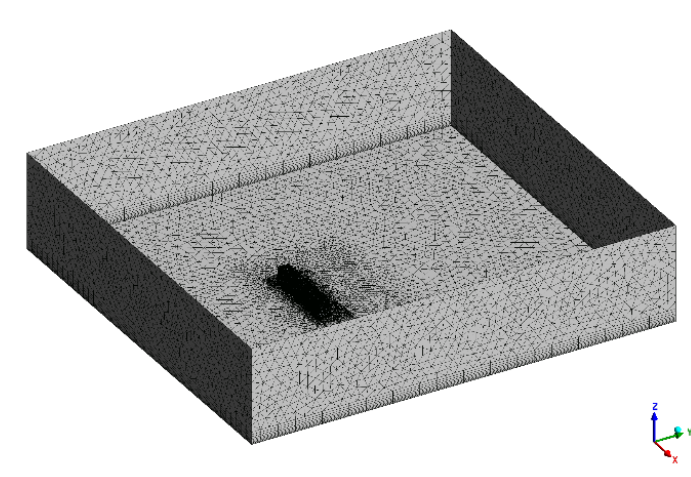

(a)

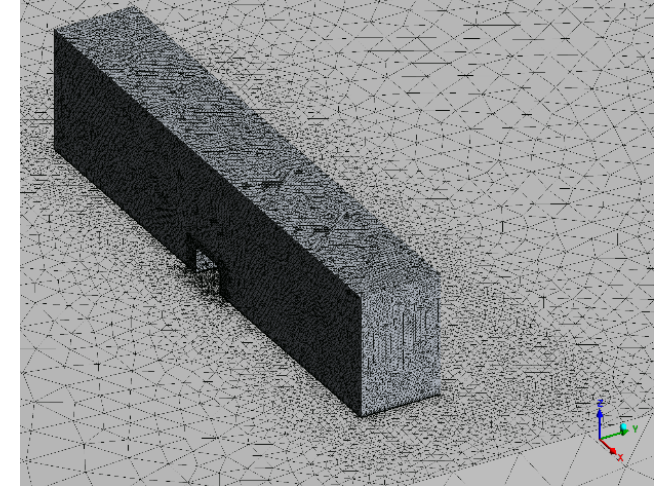

(b)

Figure 5. Computational mesh for the single-building case: (a) entire domain meshes and (b) the close view of the meshes around the building.

An incompressible transient simulation was conducted. The time-averaged velocity contour at a height of $2 \mathrm{~m}$ from the ground is shown in Figure 6. The pedestrian crossing wind speed was higher than its nearby regions due to the reductions in the cross-sectional area because of a passage at the pedestrian level. 


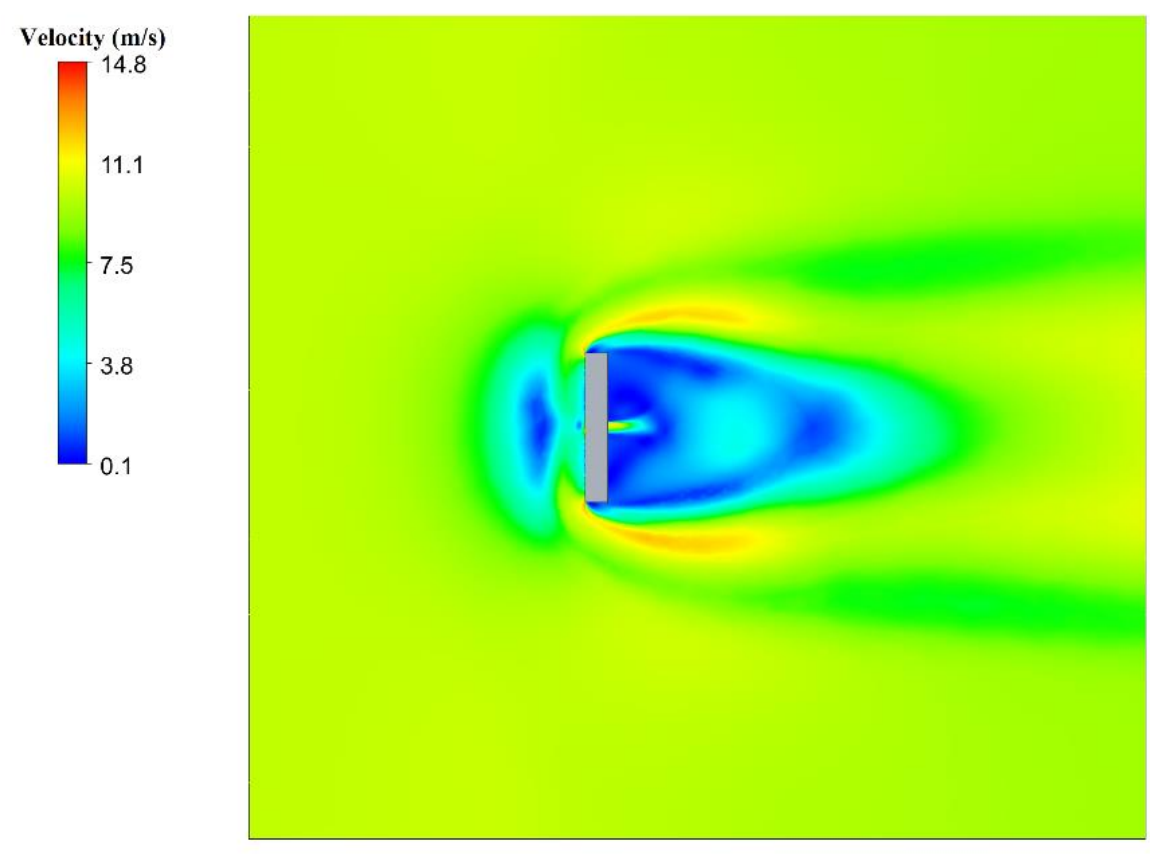

Figure 6. Flow field around the building.

Figure 7 and Table 2 show the comparison between the simulation results from different turbulence models and the experimental data. For the single building validation case, the simulation results from all the tested turbulence models showed overall similar trends to the experimental data. The realizable $k-\varepsilon$ and the second-order RSM model showcased better matches. The position of the maximum wind speed was also accurately captured compared with the wind tunnel testing results.

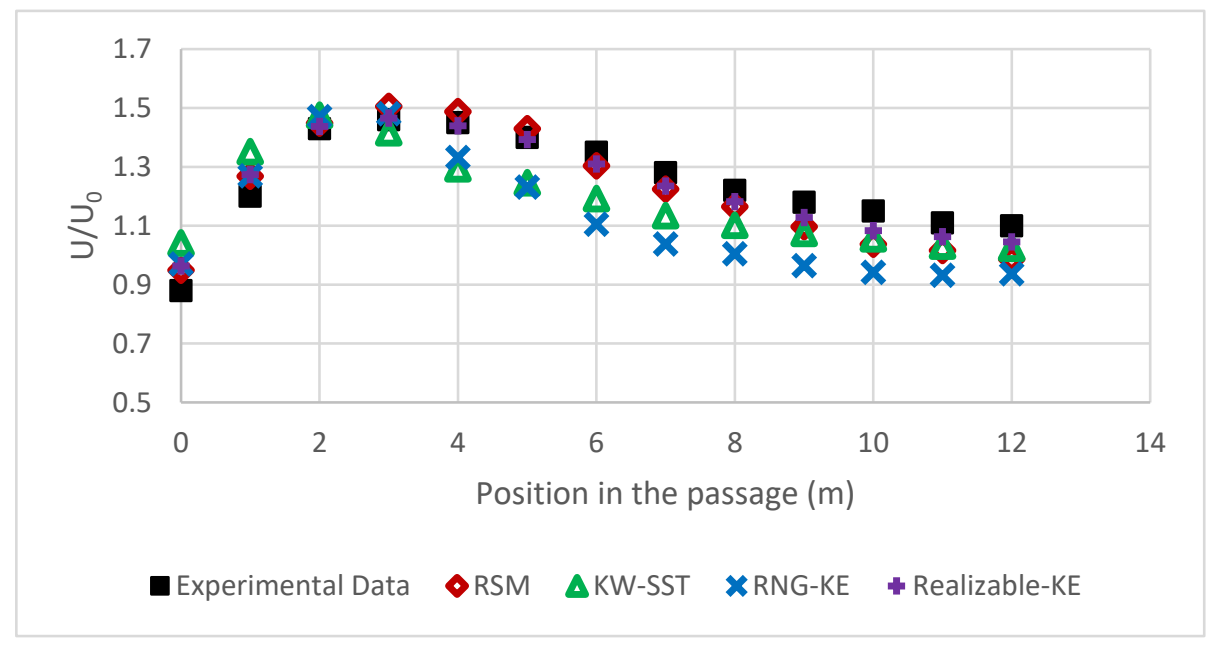

Figure 7. Comparison of the simulation results for various turbulence models and wind tunnel testing data.

Table 2. The comparison between different turbulent models for the single-building case.

\begin{tabular}{cc}
\hline Turbulence Model & Max Relative Error \\
\hline Realizable $k-\varepsilon$ & $9.42 \%$ \\
$k-\omega$ SST & $18.37 \%$ \\
RNG $k-\varepsilon$ & $18.86 \%$ \\
RSM & 10.28 \\
\hline
\end{tabular}




\subsubsection{Validation for Wind in the Two-Building Case}

Similarly, urban wind flow for the two-building case was simulated by using the RNG $k-\varepsilon$ model, realizable $k-\varepsilon$ model, $k-\omega$ SST model, and second-order RSM. The computational meshes for the entire domain are shown in Figure 8a and a close-up view of the refined meshes around the two buildings and the passage are shown in Figure 8b. Similar meshing strategies were used as in the single-building validation case. The total element number for the medium mesh was 2,242,748.

Velocity contours at the height of $2 \mathrm{~m}$ from the ground are shown in Figure 9. The stagnation region before the building, acceleration regions at the windward sides of the building, the passage, and the wake region after the buildings can all be observed clearly.

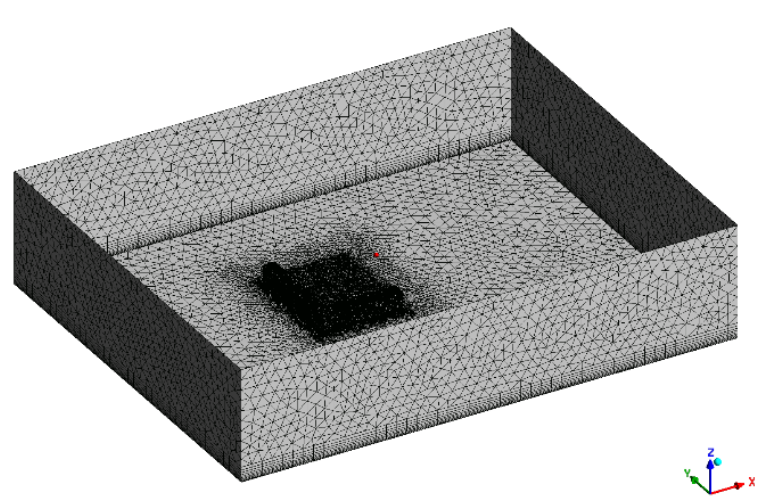

(a)

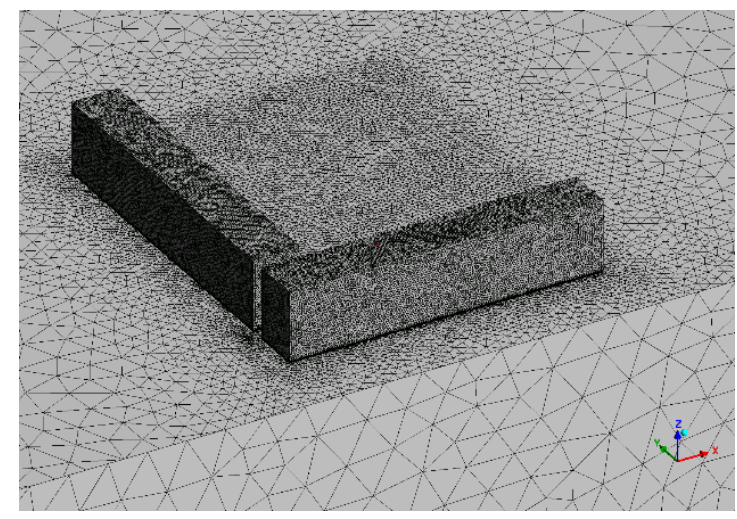

(b)

Figure 8. Computational mesh for the two-building case: (a) entire domain meshes and (b) a close-up view of the meshes around the buildings.

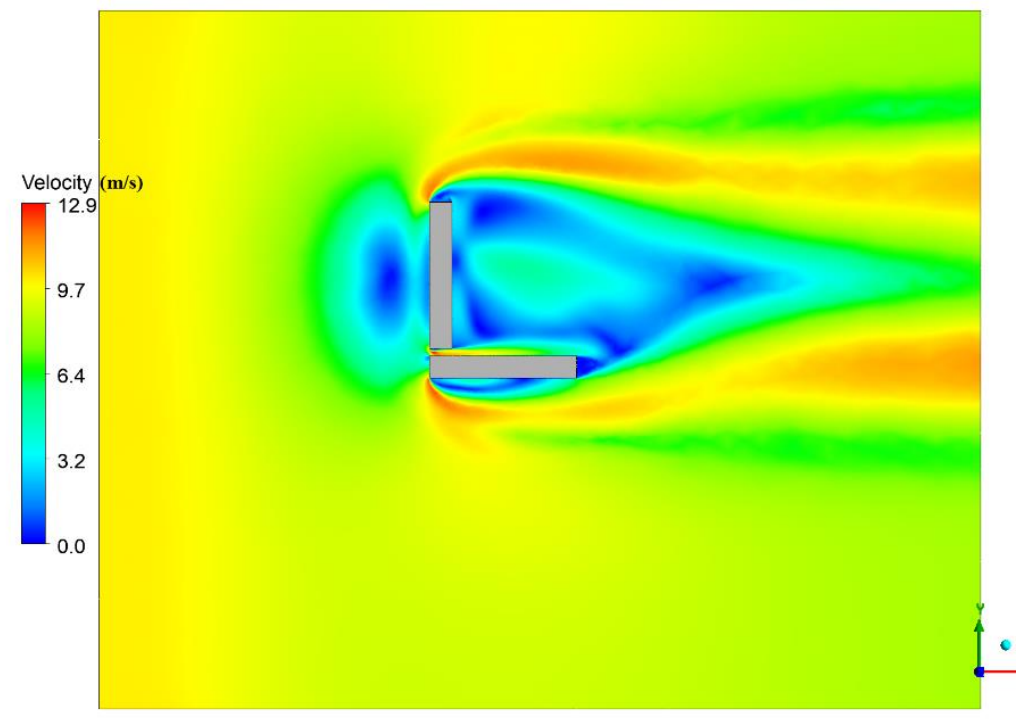

Figure 9. Flow field around the building.

The simulation results of the velocity at $2 \mathrm{~m}$ high along the central line of the passage between the two buildings were compared with experimental data [2], as shown in Figure 10 and Table 3. Except for the results obtained by using the second-order RSM, results from all other turbulence models did not show good agreement with the experimental data. 


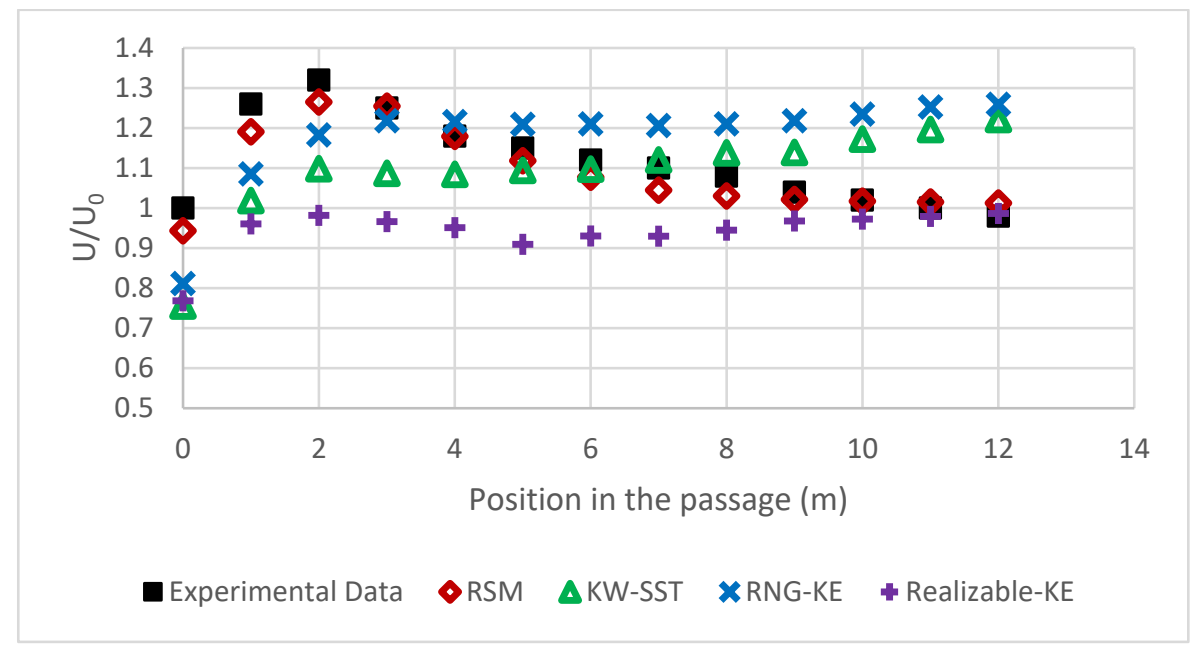

Figure 10. Comparison of the simulation results for various turbulence models and wind tunnel testing data.

Table 3. The comparison between different turbulent models for the two-building case.

\begin{tabular}{cc}
\hline Turbulence Model & Max Relative Error \\
\hline Realizable $k-\varepsilon$ & $25.62 \%$ \\
$k-\omega$ SST & $24.65 \%$ \\
RNG $k-\varepsilon$ & $28.53 \%$ \\
RSM & $5.6 \%$ \\
\hline
\end{tabular}

From the two benchmark cases, the RSM continuously showed good simulation results for wind flow in an urban environment and was therefore adopted in the following urban wind flow simulation and wind turbine siting study.

\subsection{Wind Turbine Siting in an Urban Environment}

A wind rose is a convenient method for displaying the distribution of wind direction and distribution of wind velocity in general statistical analysis. It consists of sixteen equidistant radial lines representing the direction, with the length of the line being proportional to both the frequency of the wind and the velocity of the wind from this direction. The wind rose can also show the relative wind velocity.

The data were recorded by the three sensors on the roof of the SULB penthouse. The locations of the three sensors are present in Figure 4. The overall wind rose for April 2010 to April 2011 on the PNW campus is shown in Figure 11. N, S, W, E stand for north, south, west, and east, respectively. The wind direction of the highest frequency was northeast (NE), with a wind speed magnitude of $4.16 \mathrm{~m} / \mathrm{s}$. However, when considering the year-long wind direction distribution, the dominant wind direction was from west-northwest (WNW) to south-southeast (SSE). The second most frequent wind direction, west-southwest (WSW) with a wind speed magnitude at $3.17 \mathrm{~m} / \mathrm{s}$, was applied as the input wind conditions for the following wind flow simulation. 


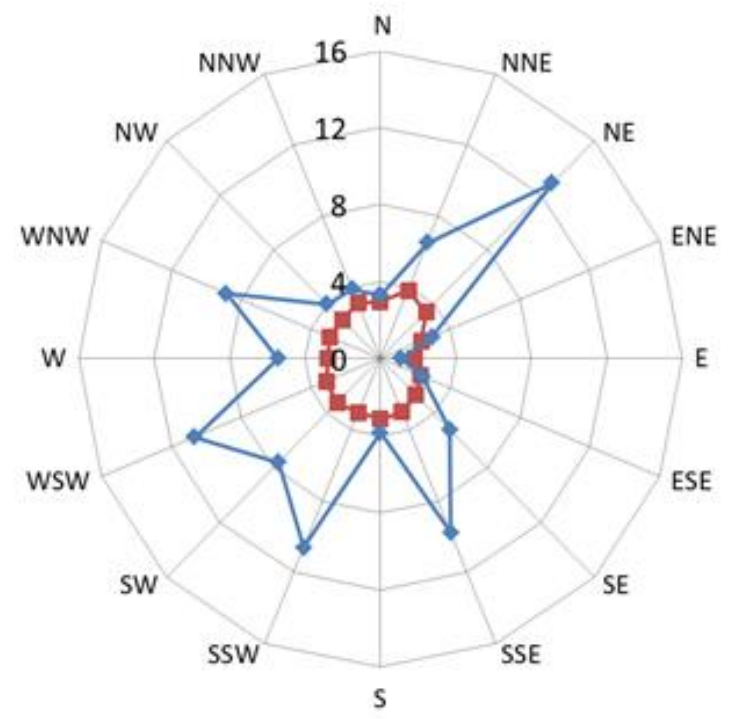

$\because$-Average Wind Speed $\rightarrow$ Frequency

Figure 11. The whole-year overall wind rose.

The average wind speed distribution for each month over the year is shown in Figure 12. April 2011 had the highest monthly average wind speed.

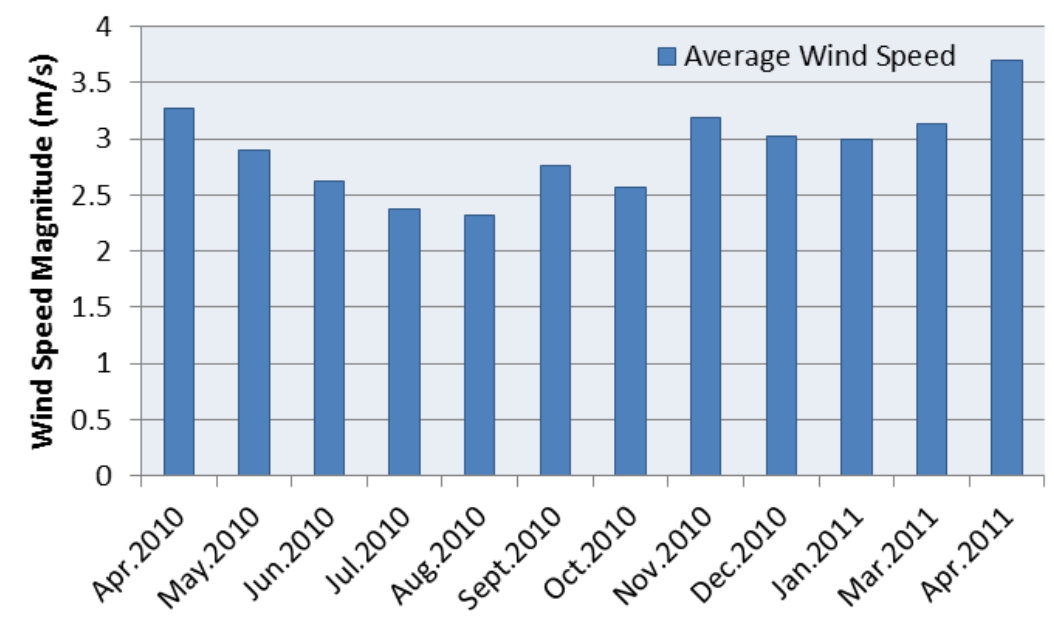

Figure 12. Monthly average wind speed distribution.

The Weibull and Rayleigh distribution results for each month over the year are shown in Figure 13. A higher probability at a higher mean wind speed can be observed for April 2011 in both the Weibull and Rayleigh distributions. 


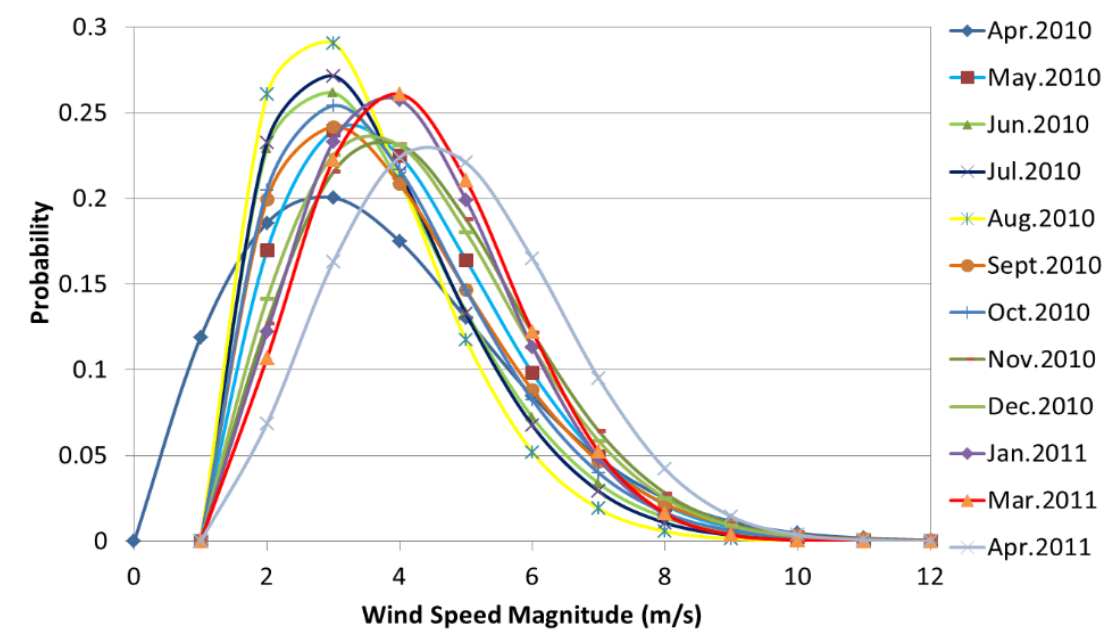

(a)

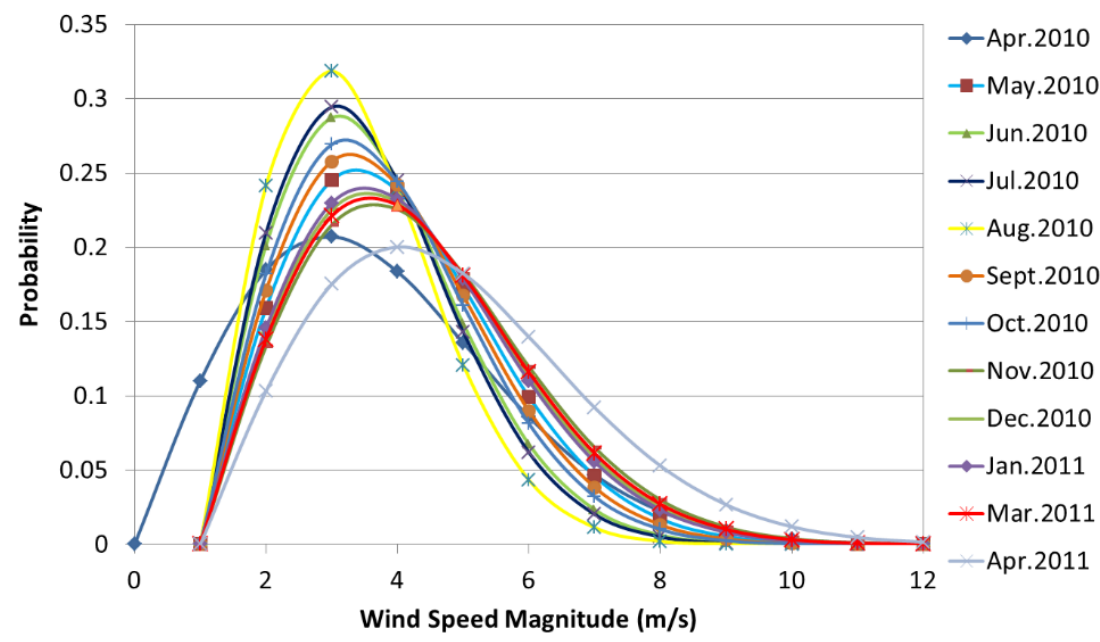

(b)

Figure 13. Statistical wind data analysis: (a) the monthly Rayleigh distribution and (b) the monthly Weibull distribution.

These statistical results were used for the wind turbine power extraction evaluation, as shown in Section 3.2.2.

\subsubsection{Urban Wind Flow Analysis}

The Eddy GT wind turbine from Urban Green Energy (New York, NY, USA) was available to be installed on the top of the PNW campus buildings' roofs. With the advantages of being lightweight and producing a low amount of noise, this small-scale vertical axis wind turbine (VAWT) has been used for residential and commercial applications in various regions in the United States. The rated power for this wind turbine is $1 \mathrm{~kW}$.

The detailed properties and performance information of that wind turbine, as stated by the manufacturer, are given in Table 4 . The total height of the turbine was $4.70 \mathrm{~m}$. 
Table 4. Eddy GT wind turbine properties and specifications.

\begin{tabular}{cc}
\hline Axis & Vertical \\
\hline Height & $2.70 \mathrm{~m}$ \\
Tower height & $2 \mathrm{~m}$ \\
No. of blades & 3 \\
Width & $1.80 \mathrm{~m}$ \\
Weight & $175 \mathrm{~kg}$ \\
Swept Area & $4.62 \mathrm{~m}^{2}$ \\
Cut-in wind speed & $3 \mathrm{~m} / \mathrm{s}$ \\
Cut-out wind speed & $30 \mathrm{~m} / \mathrm{s}$ \\
Rated wind speed & $12 \mathrm{~m} / \mathrm{s}$ \\
Survival wind speed & $55 \mathrm{~m} / \mathrm{s}$ \\
Rated power & $1000 \mathrm{~W}$ \\
\hline
\end{tabular}

In order to identify an ideal siting location for the wind turbine, three-dimensional wind fields for PNW were constructed. Based on the validation results, RSM was selected for the PNW urban wind flow simulation. As explained before, the inlet velocity profile was defined by using the $1 / 7$ power law as follows: $U=U_{0}(z / 19)^{1 / 7}, U_{0}=3.17 \mathrm{~m} / \mathrm{s}$.

Three different sets of mesh were used for the mesh-independent study, where the element numbers were 2,673,927, 7,826,295, and 12,095,887 for coarse (surface element size $0.8 \mathrm{~m}$, body element size $5 \mathrm{~m}$ ), medium (surface element size $0.5 \mathrm{~m}$, body element size $5 \mathrm{~m}$ ), and fine mesh (surface element size $0.4 \mathrm{~m}$, body element size $3 \mathrm{~m}$ ), respectively. The simulation results obtained from the medium mesh and fine mesh did not show much difference. The medium mesh was adopted in the simulation as it brought a good balance between computational accuracy and cost.

Figure 14 shows the velocity contour at a height level of $21 \mathrm{~m}$. It represents the average height of the selected turbine (half of the rotor height) on different buildings' roofs.

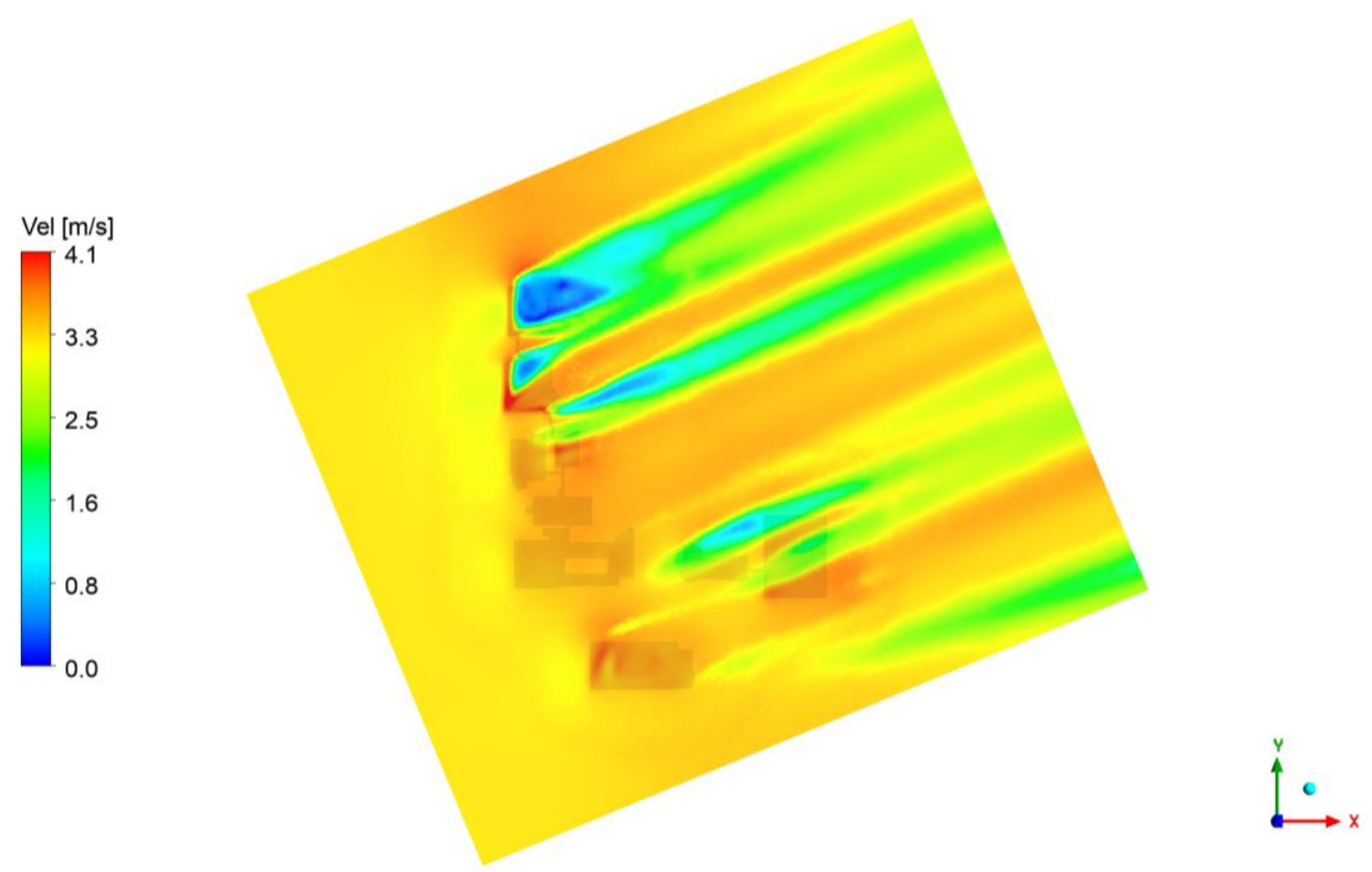

Figure 14. Contour of the wind velocity at a height level of $21 \mathrm{~m}$.

At a height level of $21 \mathrm{~m}$, the SULB showed good wind potential. Even though the CLO and Anderson buildings were higher than the SULB, at the height level of $21 \mathrm{~m}$, the wind flow above the CLO and Anderson buildings were at the lower part of the boundary layer flow region, which 
showed lower wind potential compared to the same height as the SULB. Among all the buildings on PNW's campus, the SULB can be seen as a good candidate for siting the turbine when comparing wind energy potentials.

A side view of the wind flow around the SULB is shown in Figure 15. When the wind blows over the edges of a building, the flow will separate at the leading edge of the roof at a certain angle, referred to as the skew angle. The flow was accelerated because of the separation effects. Furthermore, serving as the foundation of the wind turbine, the special penthouse structure on top of the SULB edge can also increase the wind potential due to the skew angle effects; therefore, an optimal location for wind turbine siting was proposed.

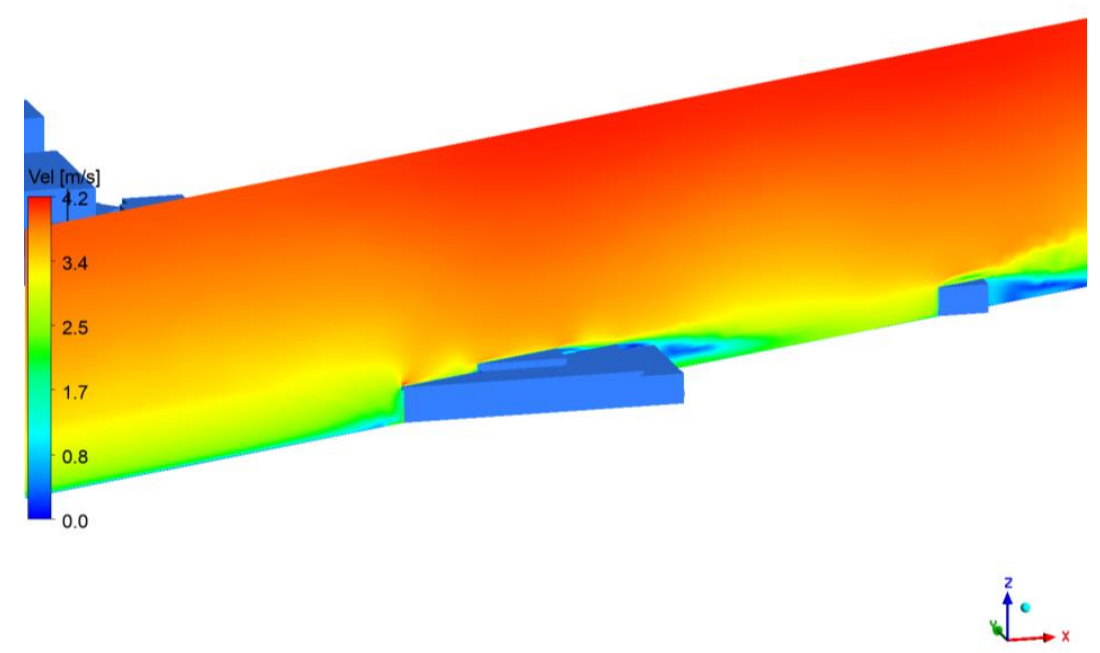

Figure 15. Contour of the wind velocity around the SULB.

After considering the simulation results, support capability of the roof structure, existing objects on top of the building roof, available budget, and overall aesthetic look, a small-scale VAWT was installed on the roof of the SULB. Figure 16a shows the final selection of the wind turbine siting location on top of the SULB, while Figure $16 \mathrm{~b}$ shows a picture of the final installation.

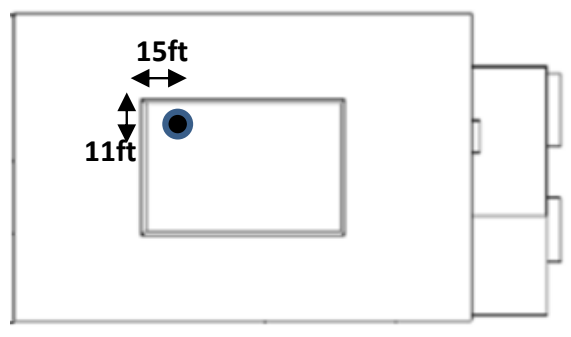

(a)

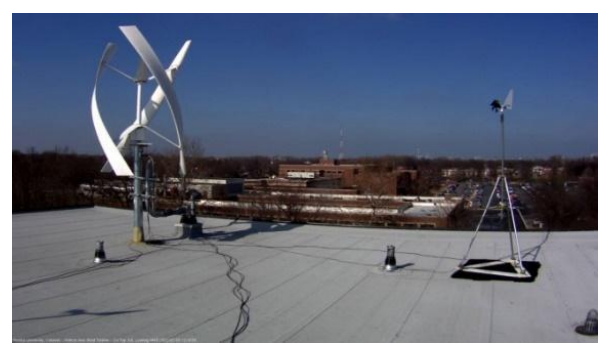

(b)

Figure 16. Wind turbine installation location: (a) suggested installation location and (b) the selected installation site.

\subsubsection{Wind Turbine Performance Analysis}

In an ideal situation, the wind turbine should be installed in a site where the rated wind speed has a high probability. Based on the wind data analysis, the wind energy potential on the PNW campus was not very high, as the annual (from April 2010 to April 2011) averaged wind speed was $2.67 \mathrm{~m} / \mathrm{s}$, which is lower than the cut-in wind speed. The Eddy GT Wind Turbine will not fully perform under this given wind condition. Over the year (April 2010 to April 2011), there were seven months that had an average wind speed higher than the cut-in wind speed. Wind power was expected to be generated 
during these periods. Due to some constraints, the wind turbine was selected even though it did not meet all the desires. In conclusion, the selection of a wind turbine is a complex decision and should be made with consideration of the wind potential, availability of resources, and budget constraints.

After the installation, a more specific analysis that focused on the wind turbine power generation was conducted.

The Eddy GT wind turbine power output curve shown in Figure 17 indicates the amount of power that could be extracted from the wind turbine at different wind speeds. The extracted power can be calculated using $P T\left(V_{w}\right)=P_{r} \frac{V_{w}{ }^{3}-V_{1}{ }^{3}}{V_{r}^{3}-V_{1}{ }^{3}}\left(V_{w}\right.$ is the wind speed, $P_{r}$ is the wind turbine rated power, $V_{1}$ is the cut-in wind speed, and $V_{r}$ is the rated wind speed) when the wind speed is greater than the cut-in speed and smaller than the rated speed. If the wind speed is greater than or equal to the rated wind speed and less than the cut-out speed, the rated power will be extracted from the wind turbine.

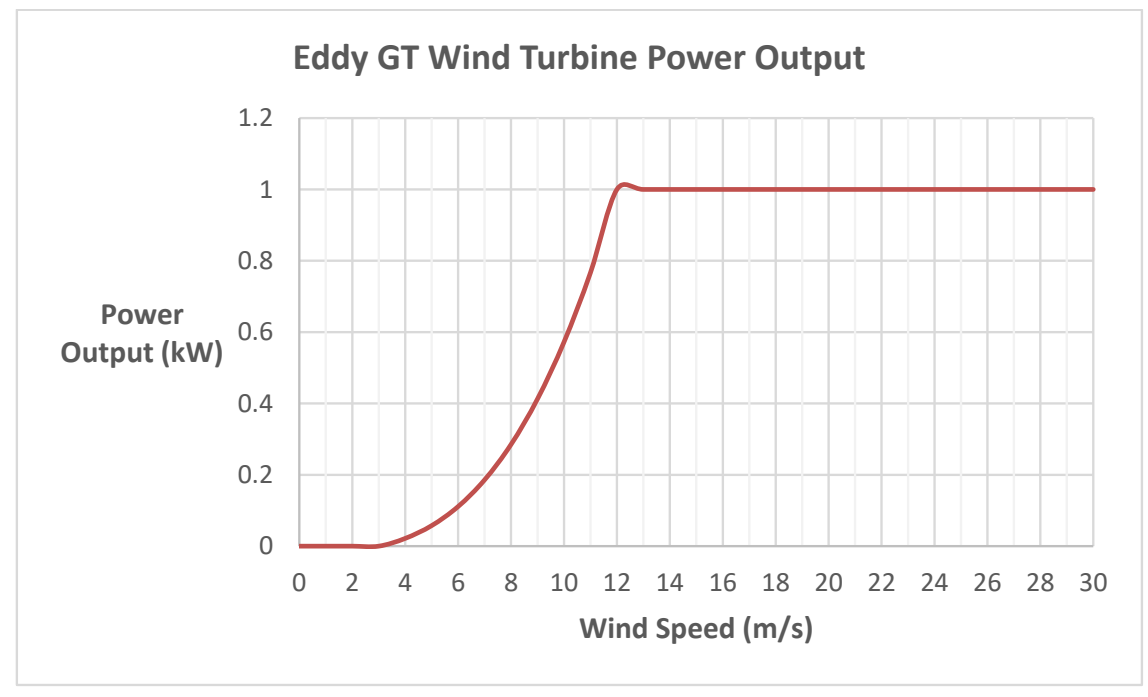

Figure 17. Eddy GT Wind Turbine power output curve.

Based on the power output curve of the wind turbine and the wind velocity probability density on the installation site, the estimated power generation of the wind turbine can be calculated. As shown in Figure 18, the total power generated over the year (April 2010 to April 2011) was $202 \mathrm{kWh}$ using the Rayleigh distribution compared to a slightly higher value of $204 \mathrm{kWh}$ obtained using the Weibull distribution. Due to the low wind energy potential in the selected urban site, the extracted power was much lower than the energy extraction at the rated power.

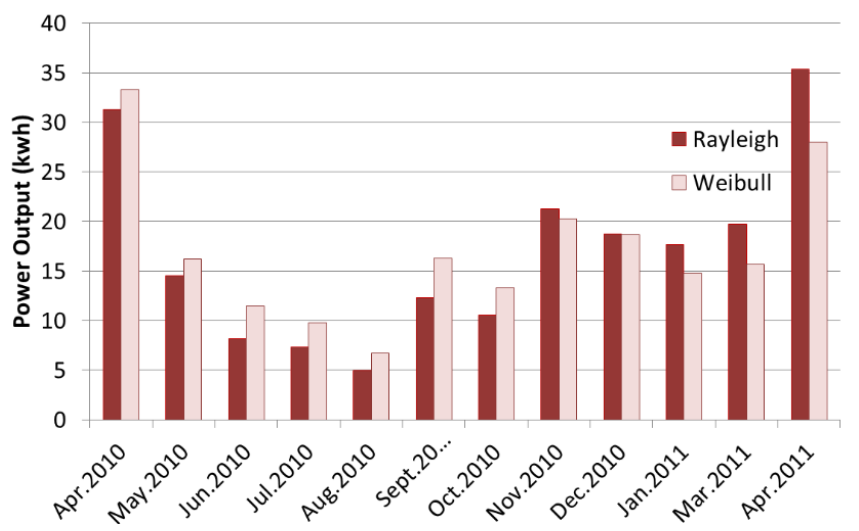

Figure 18. Monthly power generated by the Eddy GT Wind Turbine (from April 2010 to April 2011). 


\section{Discussion}

A three-dimensional CFD model was developed to simulate wind flow in urban environments. Different turbulence models were adopted to simulate urban wind flow. The simulation results were compared with wind tunnel testing data. The results showed that among all the models tested (RSM, $k-\omega \mathrm{SST}$, realizable $k-\varepsilon$, and RNG $k-\varepsilon$ models), RSM gave the best matches with experimental data in both benchmark cases. Therefore, RSM was selected to simulate the urban wind flow and wind turbine siting for Purdue University Northwest, which is located in an urban area in Northwestern Indiana.

A one-year wind data analysis result was used to set up the inlet wind profile. Statistical wind data analysis was performed using Rayleigh and Weibull distributions, and the results were used as the wind turbine energy production estimation for the selected wind turbine.

3-D suburban wind characters were studied and analyzed based on wind fields around the SULB. Flow over a building was separated at the roof windward leading edge at a certain skew angle. Wind flow became accelerated due to the flow separation, which may enhance the wind energy potential in that area. Besides serving as the foundation of rooftop wind turbines, buildings may also play an active role in helping to gain more wind energy output in an urban environment.

Furthermore, when considering the wind turbine siting on top of the building roof, a taller building has the benefit of reaching a higher wind power density. However, in order to take advantage of this feature, the selected turbine needs to reach a certain height. Otherwise, due to the lower part of the boundary layer flow effects, a relatively low building may be a better choice for a low-height wind turbine siting.

Small-scale wind turbines are usually lightweight, have low vibrations, and reduced noise. They are widely used in urban environments. Many factors need to be considered for urban wind turbine siting, such as the selected site wind potential, the probability of the wind speed compared to the cut-in wind speed and rated wind speed, the height of the wind turbine vs. the height of the buildings, the building roof structure, and existing objects. The final decision should fit within the budget limits.

Author Contributions: Conceptualization, X.W.; simulation, M.L.; validation, X.W. and M.L.; formal analysis, X.W. and M.L.; writing — original draft preparation, M.L.; writing—review and editing, X.W.; supervision, X.W. All authors have read and agreed to the published version of the manuscript.

Funding: This research received no external funding.

Acknowledgments: Special thanks are extended to Ms. Lu Lu for obtaining and process the one-year wind data collected by three wind data sensors on top of the SULB.

Conflicts of Interest: The authors declare no conflict of interest.

\section{References}

1. Stathopoulos, T.; Alrawashdeh, H.; Al-Quraan, A.; Blocken, B.; Dilimulati, A.; Paraschivoiue, M.; Pilay, P. Urban wind energy: Some views on potential and challenges. J. Wind Eng. Ind. Aerod. 2018, 178, 146-157. [CrossRef]

2. Wiren, B.G. A wind tunnel study of wind velocities in passages between and through buildings. In Proceedings of the 4th International Conference on Wind Effects on Buildings and Structures, London, UK, September 1975; pp. $465-475$.

3. Tsang, C.W.; Kwok, K.C.S.; Hitchcock, P.A. Wind tunnel study of pedestrian level wind environment around tall buildings: Effects of building dimensions, separation and podium. Build Environ. 2012, 49, 167-181. [CrossRef]

4. Weerasuriya, A.U.; Tse, K.T.; Zhang, X.; Li, S.W. A Wind tunnel study of effects of twisted wind flows on the pedestrian-level wind field in an urban environment. Build Environ. 2018, 128, 225-235. [CrossRef] [PubMed]

5. Li, J.; Peng, Y.; Ji, H.; Hu, Y.; Ding, W. A Wind tunnel study on the correlation between urban space quantification and pedestrian-level ventilation. Atmosphere 2019, 10, 564. [CrossRef] 
6. Blocken, B.; Carmeliet, J.; Stathopoulos, T. Pedestrian-level wind conditions around buildings: Review of wind-tunnel and CFD techniques and their accuracy for wind comfort assessment. Build Environ. 2016, 100, 50-81. [CrossRef]

7. Rodi, W. Comparison of LES and RANS calculations of the flow around bluff bodies. J. Wind Eng. Ind. Aerod. 1997, 69, 55-75. [CrossRef]

8. Johnson, G.T.; Hunter, L.J. Urban wind flows: Wind tunnel and numerical simulations-a preliminary comparison. Environ Modell Softw. 1998, 13, 279-286. [CrossRef]

9. Willis, R. Validation of CFD Predictions of Urban Wind. Master's Thesis, Victoria University of Wellington, Wellington, New Zealand, 2017.

10. Song, C.C.; He, J. Evaluation of pedestrian winds in urban area by numerical approach. J. Wind Eng. Ind. Aerod. 1999, 81, 295-309.

11. Santiago, J.L.; Martilli, A. CFD simulation of airflow over a regular array of cubes. Part I: Three-dimensional simulation of the flow and validation with wind-tunnel measurements. Boundary Layer Meteorol. 2007, 122, 609-634. [CrossRef]

12. Zheng, C.; Li, Y.; Wu, Y. Pedestrian-level wind environment on outdoor platforms of a thousand-meter-scale mega tall building: Sub-configuration experiment and wind comfort assessment. Build Environ. 2016, 106, 313-326. [CrossRef]

13. Gnatowska, R.; Sosnowski, M.; Uruba, V. CFD modeling and PIV experimental validation of flow fields in urban environments. E3S Web Conf. 2017, 14, 1034. [CrossRef]

14. Akashi, M.; Isaac, L. Prediction of wind environment and thermal comfort at pedestrian level in urban area. J. Wind Eng. Ind. Aerod. 2006, 96, 1498-1527.

15. Hu, K.; Cheng, S.; Qian, Y. CFD simulation analysis of building density on residential wind environment. J. Eng. Sci. Technol. Rev. 2018, 11, 35-43. [CrossRef]

16. Liu, S.; Pan, W.; Zhang, H.; Cheng, X.; Long, Z.; Chen, Q. CFD simulations of wind distribution in an urban community with a full-scale geometrical model. Build Environ. 2017, 117, 11-23. [CrossRef]

17. Reiter, S. Validation Process for CFD Simulations of Wind around Buildings. Proceedings of the European Built Environment CAE Conference. Available online: https:/scholar.google.com/scholar?hl=en\&q=Reiter+ S\%3A+\%282008\%29.+\%E2\%80\%9CValidation+Process+for+CFD+Simulations+of+Wind+Around+ Buildings\%E2\%80\%9D.+European+Built+Environment+ACE+Conference (accessed on 3 February 2019).

18. Fluent Theory Guide: Large Eddy Simulation (LES) Model. Available online: https://ansyshelp.ansys. com/account/secured?returnurl=/Views/Secured/corp/v192/flu_th/flu_th_sec_turb_les.html (accessed on 10 May 2020).

19. Fluent Theory Guide: Turbulence. Available online: https://ansyshelp.ansys.com/account/secured?returnurl= /Views/Secured/corp/v192/flu_th/flu_th_chap_turbulence.html (accessed on 10 May 2020).

20. Menter, F.R. Two-equation eddy-viscosity turbulence models for engineering applications. AIAA J. 1994, 32, 1598-1605. [CrossRef]

21. Lu, L. 3-D Wind Fields Construction for Suburban Environment. Master's Thesis, Purdue University, Hammond, Indiana, August 2012.

22. Nelson, V. Wind Energy: Renewable Energy and the Environment; CRC Press: Boca Raton, FL, USA, 2009; pp. 48-49.

23. Manwell, J.F.; McGowan, J.G.; Rogers, A.L. Wind Energy Explained: Theory, Design and Application, 2nd ed.; John Wiley \& Sons: Hoboken, NJ, USA, 2002; pp. 55-59.

(C) 2020 by the authors. Licensee MDPI, Basel, Switzerland. This article is an open access article distributed under the terms and conditions of the Creative Commons Attribution (CC BY) license (http://creativecommons.org/licenses/by/4.0/). 